\title{
Evaluation of a rapid diagnostic test for detection of SARS-CoV-2 antigen in nasopharyngeal swabs
}

\author{
Davide Carcione, ${ }^{1,2}$ Jari Intra, ${ }^{2}$ Daniela Riggio, ${ }^{1}$ Simona Sabella, ${ }^{1}$ Lorenza Rondelli, ${ }^{1}$ Simone Barbieri, ${ }^{3}$ \\ Valerio Leoni, ${ }^{3}$ Maria Luisa Biondi ${ }^{1}$
}

${ }^{1}$ Department of Laboratory Medicine, Centro Cardiologico Monzino IRCCS, Milan; ${ }^{2}$ Department of Laboratory Medicine, University of Milano-Bicocca, Azienda Socio Sanitaria Territoriale della Brianza ASST-Brianza, Desio Hospital, Desio (MB); ${ }^{3}$ Biostatistic Unit, Centro Cardiologico Monzino IRCCS, Milan, Italy

\begin{abstract}
Summary
Background and aims: Rapid and accurate diagnosis is essential to limit the spread of SARS-CoV-2 and for patient's management. Currently, real-time reverse transcription polymerase chain reaction (RT-PCR) is the recommended laboratory test to detect SARS-CoV-2. However, the requirement of special instruments and skilled personnel have limited the use of this technique. Recently, several rapid antigen detection tests have been devel-
\end{abstract}

Correspondence: Carcione Davide, Department of Laboratory Medicine, Centro Cardiologico Monzino IRCCS, Milan; Department of Laboratory Medicine, University of Milano-Bicocca, Azienda Socio Sanitaria Territoriale della Brianza ASST-Brianza, Desio Hospital, Desio (MB), Italy.

Tel.: +39.0362383400 .

E-mail: davide.carcione@asst-brianza.it

Key words: Antigen; SARS-CoV-2; COVID-19; Rapid antigen detection test.

Acknowledgments: the authors gratefully acknowledge Dr. Elena Intra for reviewing the manuscript.

Contributions: DC and JI wrote the manuscript; DC designed the study; DR, SS, and LR performed the sample analysis; SB performed the statistical analysis; VL and MLB critically revised the manuscript. All authors have accepted responsibility for the entire content of the submitted manuscript and approved submission.

Conflict of interest: the authors declare no potential conflict of interest.

Funding: None.

Received for publication: 18 January 2021.

Revision received: 10 March 2021.

Accepted for publication: 23 March 2021.

${ }^{\circ}$ Copyright: the Author(s), 2021

Licensee PAGEPress, Italy

Microbiologia Medica 2021; 36:9623

doi: $10.4081 / \mathrm{mm} .2021 .9623$

This article is distributed under the terms of the Creative Commons Attribution Noncommercial License (by-nc 4.0) which permits any noncommercial use, distribution, and reproduction in any medium, provided the original author(s) and source are credited. oped and used as frontline. The aim of this work was to assess the performances of STANDARD F COVID-19 Ag FIA Kit, a rapid fluorescence immunoassay for the detection of SARS-CoV-2 nucleoprotein antigens, in comparison to RT-PCR.

Materials and methods: Twenty-three nasopharyngeal swabs were collected and tested.

Results: Among the 20 positive RT-PCR samples, 9 were detected by the immunofluorescence assay, reporting an overall sensitivity of $45 \%$. The sensitivity increased to $64 \%$ in the case of a high viral load, where all three target genes, RdRp, N, and E, were detected by RT-PCR.

Conclusions: A better antigen detection rate is associated with low Cycle threshold values which are inversely related to the viral load. STANDARD F COVID-19 Ag test cannot be considered as the frontline assay for COVID-19 diagnosis, but it might be used in association with clinical signs of patients to reduce the number of RT-PCR testing.

\section{Introduction}

The three epidemic outbreaks caused by human coronavirus (hCoV), i.e. SARS-CoV, MERS-CoV and SARS-CoV-2, emerging at the beginning of the $21^{\text {st }}$ century, have highlighted the need for fast and accurate diagnostic assays. Initially reported in China, the last human coronavirus disease (COVID-19) caused by SARSCoV-2 has spread rapidly around the world and it has become a major public health $(1,2)$. Early diagnosis is crucial for the detection of COVID-19 infected subjects in order to control and limit the outbreak (3). The current gold-standard assay for the detection of SARS-CoV-2 is based on viral RNA amplification by using reverse transcription PCR (RT-PCR) (4). This test requires an average execution time of $2 / 3$ hours, except for GeneXpert system (Cepheid 904, USA) which can provide a result in 50 minutes, but it needs skilled personnel and it is expensive. Therefore, the evaluation of immunological diagnostic assays which can detect SARS-CoV-2 antigens at lower costs compared to molecular tests, might be helpful for a rapid and accurate diagnosis of COVID-19. Currently, there are different immunological tests that can detect SARS-CoV-2 nucleoprotein antigens. These assays are performed by using different methodologies, as enzyme-linked immunosorbent assay (ELISA), chemiluminescence immunoassay (CLIA), colloidal gold immunochromatography and fluorescent immunoassays (FIA) $(3,5,6)$. The aim of this study was to assess the performances of commercial STANDARD F COVID-19 Ag FIA Kit in comparison to molecular test for laboratory detection of SARS-CoV-2. 


\section{Materials and Methods}

\section{Clinical specimens}

We collected 23 nasopharyngeal (NP) swabs from symptomatic subjects admitted to the Monzino IRCCS Cardiological Centre, Milan, Italy, between March and July, 2020. All samples were obtained using flocked swabs with Universal Transport Medium (UTM) (Copan Diagnostics, Murrieta, CA, USA).

\section{One-step RT-PCR}

NP swabs were processed using ELITe InGenius ${ }^{\circledR}$ instrumentation (Elitech Group, France). Briefly: the first step required the extraction of the viral RNA with an ELITe InGenius ${ }^{\circledR}$ SP200 kit starting from a UTM aliquot of $200 \mu \mathrm{L}$, and the second step involved the detection of the viral genome. The amplification was performed using GeneFinder ${ }^{\text {TM }}$ COVID-19 Plus RealAMP Kit (OSANGHealthcare, Korea) which included probes that amplify three target genes of SARS-CoV-2, RNA-dependant RNA polymerase (RdRp) gene, envelope (E) gene and nucleocapsid (N) gene. Ribonuclease P (RNase P) was used as internal control (IC). Samples with SARS-CoV-2 RT-PCR Cycle threshold value $(\mathrm{Ct})$ under 43 were considered positive, according to the manufacturer's instructions. The expected time of execution is about 3 hours.

\section{Rapid SARS-CoV-2 antigen detection test}

STANDARD F COVID-19 Ag FIA Kit (SD BIOSENSOR, Inc., Republic of Korea) is a ready-to-use test which allows rapid and qualitative detection of SARS-CoV-2 nucleoproteins in nasopharyngeal swab specimens. This test, based on immunofluorescence technology, uses europium conjugated monoclonal antibody to detect SARS-CoV-2 nucleoprotein antigens. The test was carried out according to manufacturer's instruction: i) the nasopharyngeal swab specimen was inserted in an extraction tube buffer and then swirled at least five times; ii) after removing the swab, 4 drops (approximately $100 \mu \mathrm{L}$ ) of mixed sample were added in the test device. When the sample come into contact with the strip, passive diffusion allows the sample to migrate and react with the anti-SARS-CoV-2 antibodies immobilized onto the membrane and make fluorescence signal. A control line is included in the strip to assess the correct migration of the sample. The interpretation of the result is performed after $30 \mathrm{~min}$ by the STANDARD F200 Analyzer (SD BIOSENSOR, Inc., Republic of Korea), which provided a COI value (Negative $<1$ ).

\section{Statistical analysis}

Analyses were performed using SAS software package (Version 9.4 SAS Institute Inc., Cary, NC). Continuous variables are presented as mean \pm standard deviation (SD) and categorical variables as absolute numbers and percentages. Correlations between the results obtained by STANDARD F COVID-19 Ag FIA and those using GeneFinder ${ }^{\mathrm{TM}}$ COVID-19 Plus RealAMP were determined by Spearman's rank test. Receiver Operating Characteristic (ROC) curves were calculated and the area under the ROC curve (AUC) with $95 \%$ confidence interval (CI) was used to compare the ability of the STANDARD F COVID-19 Ag FIA test to confirm GeneFinder ${ }^{\text {TM }}$ COVID-19 Plus RealAMP results. Sensitivity, specificity, positive and negative predictive values (PPV, PPN) were calculated to assess the performance of STANDARD F COVID-19 Ag test with cut-off $=1(\mathrm{COI}$, cut-off index).

\section{Results}

We collected 23 nasopharyngeal swab specimens. The median age of the study population was 56 years, with a sex ratio of 3.6 (men to females). According to the molecular assay results, 20 samples were positive and 3 negative, with median $\mathrm{Ct}$ values of 38 (range: 24-46), 33 (range: 21-46), and 35 (range: 20-46) for RdRp, $\mathrm{N}$, and $\mathrm{E}$ genes, respectively. The results obtained by molecular assay were compared with those obtained by STANDARD F COVID-19 Ag antigenic test. Amongst the 23 samples, 12 specimens had concordant data with nine positive results and three negative results obtained with both detection methods. Discordant results with positive RT-PCR and negative STANDARD F COVID-19 Ag FIA assay were observed for 11 samples (55\%) (Table 1). Sensitivity and specificity of STANDARD F COVID-19 Ag FIA detection test were $45 \%$ (95\% CI, 23 to 67$)$ and $100 \%$, respectively; positive predictive value (PPV) was 1 and negative predictive value (NPV) was $0.21(95 \% \mathrm{CI}, 0$ to 0.43$)$. The area

Table 1. Results of STANDARD F COVID-19 Ag FIA Kit compared to RT-PCR in the samples tested.

\begin{tabular}{lccc} 
STANDARD F COVID-19 Ag FIA Kit & Positive (n) & $\begin{array}{c}\text { GeneFinder } \\
\text { PIM COVID-19 Plus RealAMIP Kit }\end{array}$ & Total (n) \\
Positive & $9(45 \%)$ & $0(0 \%)$ & 9 \\
Negative & $11(55 \%)$ & $3(100 \%)$ & 14 \\
\hline Total & 20 & 3 & 23 \\
\hline
\end{tabular}

Table 2. Results of the STANDARD F COVID-19 Ag FIA Kit compared to RT-PCR in the samples tested according to target genes detection.

\begin{tabular}{|c|c|c|c|c|c|}
\hline \multirow{2}{*}{ STANDARD F COVID-19 Ag FIA Kit } & \multicolumn{5}{|c|}{ GeneFinder ${ }^{T M}$ COVID-19 Plus RealAMP Kit: target genes (n) } \\
\hline & $\mathrm{N}(\%)$ & $N+E(\%)$ & $\mathrm{RdRp}+\mathrm{N}+\mathrm{E}(\%)$ & Negative (\%) & Total \\
\hline Negative & $5(100)$ & $2(50)$ & $4(36)$ & $3(100)$ & 14 \\
\hline Positive & $0(0)$ & $2(50)$ & $7(64)$ & $0(0)$ & 9 \\
\hline Total & 5 & 4 & 11 & 3 & 23 \\
\hline
\end{tabular}

RNA-dependant RNA polymerase $(R d R p)$ gene, envelope $(E)$ gene and nucleocapsid $(N)$ gene. 
under the curve was $0.85(95 \%$ CI $0.76-0.94$, $p<0.0001)$. Out of the eleven discordant samples resulted positive using RT-PCR, four were positive for the detection of all three target genes RdRp, N, and $\mathrm{E}$, two were positive for the detection of target genes $\mathrm{E}$ and $\mathrm{N}$, and five for only the target gene N (Table 2). On the other hand, among the nine concordant specimens, two samples were positive for the detection of target genes $\mathrm{N}$ and $\mathrm{E}$ (median $\mathrm{Ct}$ of 33 and 35, respectively), and seven for the detection of all three target genes RdRp, N, and E (median $\mathrm{Ct}$ of 25, 23 and 22, respectively). Comparing the results between the two assays, we noted that a positive rapid test result was obtained if more target genes were detected by RT-PCR. Particularly, if the RdRp target gene was detected, the concordance increased up to $64 \%$ (Table 2).

We performed a correlation analysis between rapid test results and cycle thresholds of target genes RdRp, E, and N. The samples detected as positive by the rapid antigenic assay fully correlated with the molecular results, which presented Ct RdRp, N and E values less than 30 (Figure 1). No negative results were obtained by the molecular test with $\mathrm{Ct}$ target gene values less than 30. Eleven samples resulted negative using the rapid antigenic assay but positive using the molecular test presented median $\mathrm{Ct}$ values of 42, 35, and 40 for RdRp, N, and E genes, respectively.

Moreover, we analyzed the correlation between COI values of rapid test and cycle thresholds of target genes RdRp, E, and N. $\mathrm{COI}$ values greater than 1 considered as positive by antigenic test fully correlated with the molecular positive results, which presented Ct RdRp, $\mathrm{N}$ and $\mathrm{E}$ values less than 30. Eleven samples resulted negative using the rapid antigenic assay (median COI values of 0.43 ) but positive using the molecular test presented median $\mathrm{Ct}$ values of 42,35 , and 40 for RdRp, N, and E genes, respectively. The three samples resulted negative using the molecular test (median $\mathrm{Ct}$ values of 46) presented a median COI value of 0.33 using the rapid antigenic test.

\section{Discussion}

In the current pandemic context of COVID-19, diagnostic testing for SARS-CoV-2 is necessary to identify positive subjects among the population and limit the spread of the virus. An accurate and rapid diagnosis is followed by a correct management of the infected subjects. Several companies have developed rapid tests for both antibodies and antigens detection of SARS-CoV-2 (3). The fluorescent immunoassay STANDARD F COVID-19 Ag FIA might represent one of the aforementioned assays for the laboratory detection of SARS-CoV-2 nucleoprotein antigens. Although this test presents several advantages, such as the ease and speed of execution, the reduced impact on instrumentation, the lower cost, the absence of skilled personnel, compared with molecular assays, data showed here suggested that this immunofluorescent assay is suffering from poor sensitivity. This rapid test detected SARS-CoV2 nucleoprotein antigens in nasopharyngeal specimens with $\mathrm{Ct}$ less than 30 , a result that is presumably related to a high viral load, but the sensitivity decreases in the case of the detection of target genes with $\mathrm{Ct}$ greater than 30 , most probably due to a low viral load. In our study, the specificity was $100 \%$, while the overall sensitivity of STANDARD F COVID-19 Ag FIA was 64\% if we considered the positive samples presenting the molecular detection of all target genes. This low sensitivity was already observed in other rapid diagnostic tests for SARS-CoV-2 and in those during Influenza A (H1N1) pandemic (7-9). A limit of the study is that negative samples

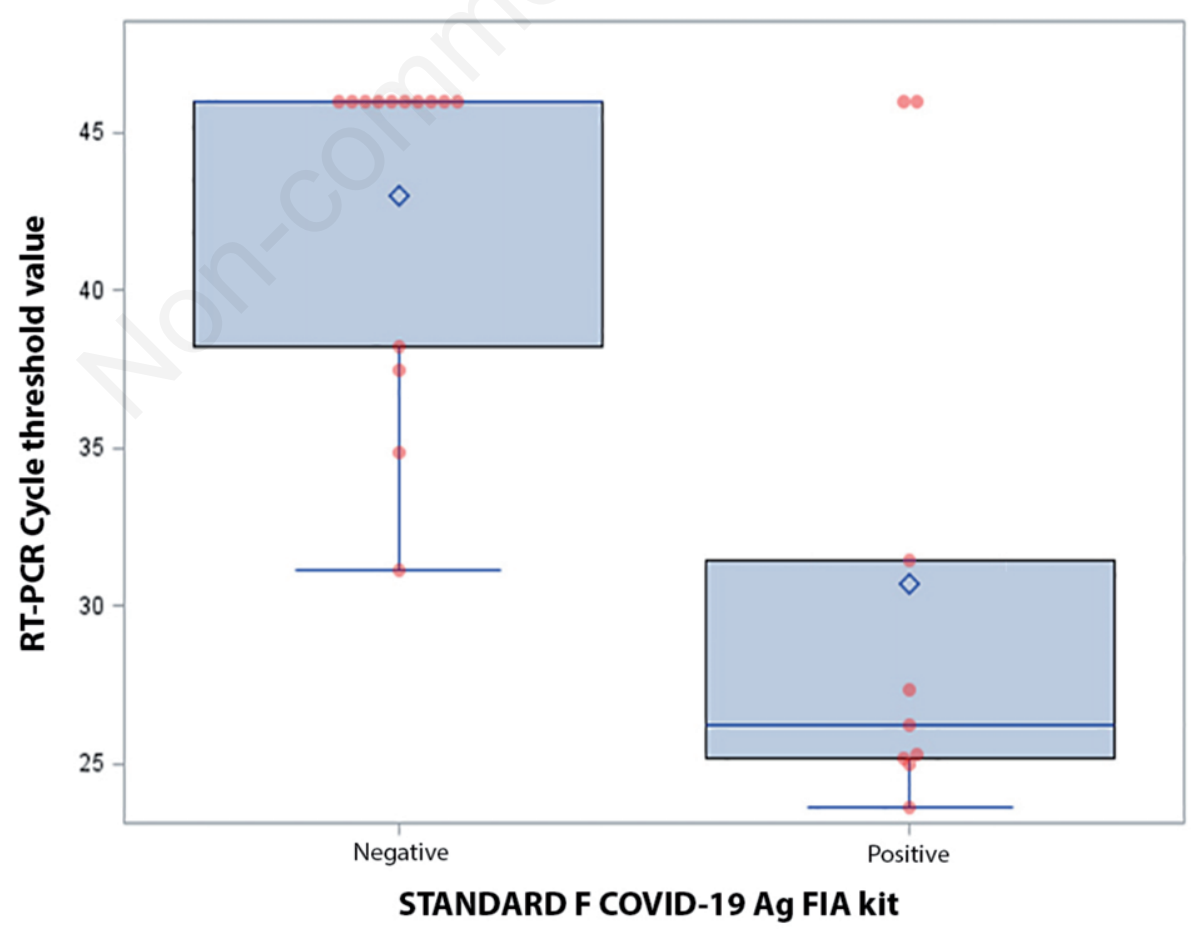

Figure 1. RT-PCR Cycle threshold of RdRp gene for STANDARD F COVID-19 Ag positive and negative tests in studied samples. Values shown are median (Blue line at the top of box in negative result, blue line within box in positive result), $25^{\text {th }}$ and $75^{\text {th }}$ percentiles (bottom and top of box, respectively), and mean (open diamond). Red circles represent the Ct values of samples. Similar results were obtained for $\mathrm{N}$ and $\mathrm{E}$ target genes (data not shown). 
are only three. A larger number of subjects are needed in order to verify and improve our results.

\section{Conclusions}

Our data showed that the STANDARD F COVID-19 Ag test cannot be considered as a frontline assay to rule-out negative subjects, but it might be used for rapid SARS-CoV-2 detection in endemic area and following European Centre for Disease prevention and Control (ECDC) and Istituto Superiore Sanità (ISS) guidance's on the appropriate use of antigenic tests both for people with or without symptoms $(10,11)$.

\section{References}

1. Huang C, Wang Y, Li X, et al. Clinical features of patients infected with 2019 novel coronavirus in Wuhan, China. Lancet 2020;395:497-506.

2. Zhang G, Zhang J, Wang B, et al. Analysis of clinical characteristics and laboratory findings of 95 cases of 2019 novel coronavirus pneumonia in Wuhan, China: a retrospective analysis. Respir Res 2020;21:74.

3. Mathuria JP, Yadav R, Rajkumar. Laboratory diagnosis of SARS-CoV-2 - A review of current methods. J Infect Public Health 2020;13:901-5.

4. World Health Organization. Laboratory testing strategy recom- mendations for COVID-19: interim guidance, 21 March 2020. World Health Organization. https://apps.who.int/iris/handle/ $10665 / 331509$.

5. Cerutti F, Burdino E, Milia MG, et al. Urgent need of rapid tests for SARS CoV-2 antigen detection: Evaluation of the SDBiosensor antigen test for SARS-CoV-2. J Clin Virol 2020;132:104654.

6. Dinnes J, Deeks JJ, Adriano A, et al. Rapid, point-of-care antigen and molecular-based tests for diagnosis of SARS-CoV-2 infection. Cochrane Database Syst Rev 2020;8:CD013705.

7. Centers for Disease Control and Prevention (CDC). Evaluation of rapid influenza diagnostic tests for detection of novel influenza A (H1N1) Virus - United States, 2009. MMWR Morb Mortal Wkly Rep 2009;58:826-9.

8. Lambert-Niclot S, Cuffel A, Le Pape S, et al. Evaluation of a Rapid Diagnostic Assay for Detection of SARS-CoV-2 Antigen in Nasopharyngeal Swabs. J Clin Microbiol 2020;58:e00977-20.

9. Scohy A, Anantharajah A, Bodéus M, et al. Low performance of rapid antigen detection test as frontline testing for COVID19 diagnosis. J Clin Virol 2020;129:104455.

10. European Centre for Disease Prevention and Control (ECDC). Options for the use of rapid antigen tests for COVID-19 in the EU/EEA and the UK. 2020; Available from: Options for the use of rapid antigen tests for COVID-19 in the EU/EEA and the UK (europa.eu).

11. Ministero della Salute Istituto Superiore di Sanità Nota tecnica ad interim Test di laboratorio per SARS-CoV-2 e loro uso in sanità pubblica. Available from: https://www.iss.it/documents/20126/0/COVID+19_+test+v4k_last.pdf/9ab1f2117d88-bcb1-d454-cfed04aa8b05?t=1604483686312. 